\title{
Observations sur quelques stades d'évolution d'Oribates récoltés au Tchad
}

\author{
par J. GRUVEL et M. GRABER \\ Laboratoire de Farcha-Fort-Lamy, Tchad
}

\begin{abstract}
RÉSUMÉ
La recherche du cycle évolutif des Cestodes du mouton du Tchad a conduit à étudier les Acariens Oribatides considérés comme hôtes intermédiaires dans la transmission de ces Helminthes.

Le principe d'élevage développé par allleurs, est rappelé brièvement. Cinq espèces d'Oribates, parmi les plus fréquentes, ont été mises en élevage ; trois ont donné des cycles de reproduction complefs dont chaque stade a été observé.

Ces trois espèces appartiennent aux familles des :

Cérotozetidae.

Scheloribatidoe.

et Galumnidae.

Les trois cycles examinés ont en commun, un stade larvaire très bref ( 1 jour), trois stades nymphaux d'une durée de 12 jours environ, une éclosion de l'adulte qui sort de l'exuvie par un mouvement de recul, la déhiscence étant du type circumgastrique. La durée totale des stades intermédiaires est de 13 jours. Deux générations ont pu être cinsi observées en 4 mois.

Ces premières observations montrent la possibilité d'un élevage complet au Laboratoire des Oribates. Des études ustérieures doivent permettre de préciser lous les éléments de la reproduction de ces Acariens.
\end{abstract}

Bıbliographie : 7 références.

La recherche du cycle évolutif des Cestodes du mouton du Tchad a conduit à étudier les Acariens Oribates, consıdérés comme hôtes intermédiaires dans la transmission de ces Helminthes.

Cinq espèces d'Oribates (les plus fréquentes) ont été mises en élevage au Laboratoire dans des conditions précisées par ailleurs : mrlieu de terre chauffée à $180^{\circ} \mathrm{C}$ pendant 1 heure, rondelles de pomme de terre assurant la source de nourriture et d'humidité, plèce maintenue entre 24 et $28^{\circ} \mathrm{C}$. Sur les cinq espèces élevées, trois seulement ont donné des cycles de reproduction complets. Elles appartiennent aux familles de: Cératozetidae, Schelorıbatıdae et Galumnidae.

\section{Cycles évolutıfs des Oribates en élevage au Laboratoire}

$1^{\circ}$ Espèce de la Fom. Ceratozetidae :

Oribates globuleux, brun foncé, portant parfois la partie antérieure du notogaster une petite zone circulaire plus claıre bordée latéralement de 2 petits points rouges. Ces Oribates sont très actifs et n'ont pas tendance à se grouper pour former des clans. L'élevage, commencé dès le 9 novembre par 86 adultes originaires de l'arbre 10 , se continue 4 mois après et a durant cette pérıde donné 2 générations : l'une dans la première quinzaine de décembre, l'autre dans la deuxième quinzaıne de janvier. 
L'œuf est ovoide, allongé, d̀ coquille bien nette, long de 210 d̀ 220 microns.

La larve est blanche, trapue, bombée dorsalement et porte de longs poils dressés (trois fois environ la longueur du corps).

Les trois stades nymphaux, à morphologie identique à celle de la larve, se différencient par la taille.

L'adulte émerge en marche arrière de la dernière mue, par une déhiscence postérieure et présente aussitôt une très forte activité. Sa coloration très pâle à l'éclosion, fonce très rapidement dans les heures qui suivent la naissance. La fécondité est suffisante pour assurer la constance de la population.

$2^{\circ}$ Espèce de la Fam. des Scheloribatidae. Espèce type :

Scheloribates perforatus.

Oribates allongés, ovoïdes, globuleux, très foncés, actifs, avec une tendance à vivre groupés.

L'élevage dure depuis 6 semaines et a donné déjà un cycle complet de reproduction :

_- l'œuf est ovoïde, allongé, long de $220 \mathrm{mi}-$ crons ;

- la larve esł piriforme, très allongée, blanc jaunâtre, dépourvue de longs poils, les pattes brun foncé, sont placées très antérieurement.

- nymphes : trois stades nymphaux, représentés par 3 tailles différentes, morphologiquement identiques à la larve.
- l'adulte s'extrait de la mue par une fente postérieure et prend aussitôt son activité.

$3^{\circ}$ « Galumno baloghi » WALL. (Fam. Galumnidé).

Originaires du bord du Chari, mis en élevage fin décembre, ont déjà donné une reproduction complète.

— œuf : long de 189 microns, sub. sphérıque ;

-- larve : très petite, blanchâtre, légèrement allongée, globuleuse et lisse; les pattes sont fortement testacées ;

- nymphe : 3 stades nymphaux de tailles croissantes, présentant les mêmes caractéristiques que la larve :

- l'éclosion se fait comme chez les espèces ci-dessus, par un recul de l'adulte qui sort de la mue par une fente horizontale postérieure.

\section{CONCLUSIONS}

Ces trols cycles de reproduction ont en commun :

- 1 stade larvarre très court ;

- 3 stades nymphaux plus longs ;

- 1 éclosion de l'adulte qui s'extrait de la mue par une marche arrière ; la fente de sortie postérieure ef horizontale se prolongeant jusqu'aux régions antérieures.

\section{SUMMARY}

\section{Observations on some stages of evolution of oribatids collecied in Chad}

Research into the life cycle of Cestodes of the sheep in CHAD has led to the study of the Oribatid mifes considered to be intermedıate hosts in the fransmission of these Helminths.

The system of propagation of the mites which has also been developed is recalled briefly. Five species of Oribatids, among the most frequent, have been bred, three have completed their reproductive cycles, each phase of which has been observed. These three species belong to the families Ceratozetidae, Scheloribatidae and Golumnidae.

The three species examined have in common, a very short larval stage (One day) and three nymphal stages which last about 12 days. The adult leaves its exuviae by a backward movement, the dehiscence being circumgastric. The total duration of the intermediate stages is 13 days. Two generations may thus be observed in 4 months.

These pretiminary observation demonstrate the possibility of Laboratory propagation of Oribatids. Further investigations should clarify all the elements of reproduction of these mites.

Bibliography : 7 references. 


\section{RESUMEN}

Observaciones en algunos estadios de evalucrón de los Oribatos recogidos en el Tchad

La busqueda del ciclo de evolución de los céstodos de la oveja en el Tchad condujo a estudiar los acáridos Oribatidos considerados como huéspedes intermediarios en la transmisión de estos helmintos.

Se recuerda brevemente el principio de crianza, desarollado por otro lado. Se criaron cinco especies de Oribatos, eníre las más frecuentes. Tres presentaron ciclos de reproducción completos cuyos diferentes estadios fueron observados.

Estas ires especies pertenecen a las familias de las :

- Ceratozetidae.

- Scheloribatidae.

- Galumnidae.

Los tres ciclos observados tienen en común : un estado de larva muy breve (1 dia), tres estados de ninfa con una duración de casi 12 días, un nacimiento del adulio que sale de la exuvia con un movimiento de retroceso, la dehiscencia siendo del tipo circungástríco. Es de 13 días la duración completa de los esiadios intermediarios. De esia manera se pudo observar dos generaciones durante 4 meses.

Estos primeras observaciones muesiran la posibilidad de una crianza completa en el laboralorio de los Oribatos. Estudios ulteriores deben permilir de dejerminar todos los elementos de la reproducción de estos acáridos.

Bibliografía : 7 referencias.

\section{BIBLIOGRAPHIE}

BAKER (E. W.) et WHARTON (G. W.). - An Introduction to Acarology, Mac Millan company. New York, 1952.

GRANJEAN (F.). - Observations sur les Oribates (18e série). Bull. Mus. Not. Hist. Not. Paris, 1947, 2 (19) : 395-402.

GRANJEN (F.). - Scheloribatidae ef Oribatulidae. Bull. Mus. Nat. Hist. Nat. Parls, 1958, 30 (4) : 352-59.

SENGBUSH (H. G.). - Studies on the life history of three Oribatid mites with observations on other species. Ann. Ent. Soc, Amer., 1954. $47:$ 646-67.

SOLDAVOTA (A. P.) . - Contribution to the study of the biology of oribatid mites. R. AC. SC. U.R.S. S., 1945, 46 (8) : 343-4.

STUNKARD $(\mathrm{H}$. W. $)$. -- Studies on the life history of the Oribatid mites. Anot. Rec., 1944, $89: 550$.

WOODRING (J. P.) et COOK (E. F.). - The biology of Ceratozetis cesalpinus Berl., Scheloribates laevigatus K., Offia neerlandica Oudms; with a description of all stages. Acorologia, 1962, 4 (1) : 101-37. 